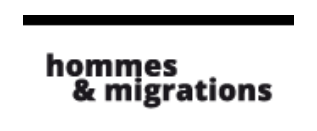

\section{Hommes \& migrations}

Revue française de référence sur les dynamiques

migratoires

$1283 \mid 2010$

Cuisines et dépendances

\title{
Vivre le Fouta à Mantes-la-Jolie
}

De la déterritorialisation à la reterritorialisation des pratiques

alimentaires chez les migrants originaires de la vallée du fleuve Sénégal

\section{Mohamadou Sall}

\section{(2) OpenEdition}

\section{Journals}

Édition électronique

URL : http://journals.openedition.org/hommesmigrations/991

DOI : 10.4000/hommesmigrations.991

ISSN : 2262-3353

Éditeur

Musée national de l'histoire de l'immigration

Édition imprimée

Date de publication : 1 janvier 2010

Pagination : $62-72$

ISBN : 978-2-919040-04-9

ISSN : $1142-852 X$

Référence électronique

Mohamadou Sall, «Vivre le Fouta à Mantes-la-Jolie », Hommes \& migrations [En ligne], 1283 | 2010,

mis en ligne le 29 mai 2013, consulté le 01 mai 2019. URL : http://journals.openedition.org/

hommesmigrations/991 ; DOI : 10.4000/hommesmigrations.991 


\section{Vivre le Fouta à Mantes-la-Jolie \\ De la déterritorialisation à la reterritorialisation des pratiques de la vallée du fleuve Sénégal} alimentaires chez les migrants originaires

Par Mohamadou Sall, démographe, enseignant-chercheur à l'Institut des sciences de la population et du développement (ISPD), université Cheikh Anta Diop de Dakar (UCAD)

Du Fouta sénégalais aux immeubles de Mantes-la-Jolie, les populations originaires du nord du Sénégal réinventent leur art culinaire. Loin de leur pays d'origine, elles ont à préserver, adapter puis transmettre leurs pratiques alimentaires et les modes de sociabilité qui les accompagnent. Autour du partage du repas, c'est un espace symbolique qu'il s'agit de faire vivre, l'espace d'une mémoire individuelle et collective

tendue vers l'Afrique. Garante de la préservation de l'identité, cette mémoire des noms de produits, des goûts et des plats permet de renouveler ses racines dans un environnement culturel différent. 
Dans le milieu de la diaspora sénégalaise en France, Mantes-la-Jolie, située dans le département des Yvelines, est considérée comme le prolongement des villages de la vallée du fleuve Sénégal en France. Certains migrants lui donnent le nom de "Fouta Faraynsé", littéralement le "Fouta de la France", en référence à cette région septentrionale du Sénégal. Cette désignation serait dictée par des faits démographiques, sociaux et culturels. D'un point de vue démographique, Mantes-la-Jolie abrite une forte communauté sénégalaise, originaire en particulier du Fouta. Elle partagerait ces particularités avec la localité des Mureaux. Les premiers migrants originaires du Fouta étaient des ouvriers venus travailler dans l'usine automobile de Renault Flins. Il s'agissait d'une migration sélectionnée, essentiellement masculine, avant que les hommes ne fassent venir leurs épouses, créant les conditions d'une reproduction biologique et sociale au niveau local.

La communauté halpular (peuhle) de Mantes-la-Jolie, très active, est représentée au niveau du conseil municipal. Elle est à l'origine de projets de coopération décentralisée développés avec des villages du Fouta. La désignation de "Fouta Faraynsé" serait aussi dictée par la reproduction sur place des modes de vie des villages d'origine. Dans cette reproduction des modes de vie, les pratiques alimentaires constituent un maillon essentiel.

\section{Présentation de l’approche méthodologique}

À Mantes-la-Jolie, dix femmes ont été interrogées, afin de mieux appréhender la consommation et la préparation (mode de cuisson, ustensiles utilisés) de plats de leurs terroirs d'origine, le rôle de l'alimentation dans la socialisation et ses formes prises les jours de fêtes. Le choix des femmes s'explique par le fait qu'elles sont traditionnellement responsables de l'alimentation au sein du ménage. Dans la tradition sénégalaise en général, et peuhle en particulier, c'est l'homme qui pourvoit aux besoins économiques du ménage. C'est lui qui "donne la dépense", c'est-à-dire qui fournit la somme nécessaire pour préparer les repas du jour. La femme est chargée d'ajuster, de réajuster "la dépense", d'acheter les denrées et de préparer les repas. Ce rôle traditionnel des femmes ne semble pas connaître de changements pour les ménages d'origine peuhle installés à Mantes-la-Jolie. La femme est toujours au centre de l'alimentation domestique. En référence à cette femme, Isabelle Garabuau-Moussaui a écrit La mère : une figure emblématique de cuisine $e^{(1)}$ ".

Deux commerçants ont aussi été interrogés sur leur village d'origine, leur date d'arrivée en France, la date de début de l'activité commerciale et leur lieu de vente. 
Enfin, douze personnes vivant au Sénégal mais ayant séjourné à Mantes-la-Jolie ont été interrogées sur la période et la raison de leur séjour en France (migration de travail, de loisirs, stage, études, etc.) et sur le statut de résident à Mantes-la-Jolie (visiteur occasionnel, résident saisonnier, résident permanent). L'accueil intégrant systématiquement dans la tradition pular une dimension alimentaire, on a recueilli auprès d'eux, via un guide d'entretien, des informations sur cette dimension, ainsi que sur les moments de convivialité autour des repas.

\section{Du Fouta à Mantes-la-Jolie : une continuité des pratiques alimentaires}

On peut établir une typologie des pratiques alimentaires au Sénégal qui coïncident avec les aires culturelles ethniques du pays. Les Diolas, au Sud, ont une alimentation dans laquelle le riz et l'huile de palme demeurent les composants de base. Au centre et au nord du Sénégal, les Peuhls ont une alimentation traditionnelle à base de céréales. Le repas de midi est souvent du gniri, un plat à base de pâte de mil cuite avec de l'eau, du poisson frais ou séché et des condiments. Le plat du soir consiste en du couscous de mil accommodé à plusieurs sauces.

À Mantes-la-Jolie, les populations pular d'origine sénégalaise ont gardé leurs habitudes alimentaires. Le repas de midi est toujours à base de riz, cuit et assaisonné différemment ou accompagné de plusieurs sauces. Ainsi, le plat peut être du riz au poisson, du riz à la viande de mouton ou de bceuf, du riz au poulet. Dans toutes ces préparations, on fait revenir le riz pour le cuire dans la sauce de poisson, de viande ou de poulet.

La seconde variété de plats est composée de riz cuit séparément, d'abord à la vapeur puis dans un bouillon : c'est le gnankatang. Il est servi avec plusieurs types de sauces : sauce à la viande et au beurre de cacahuètes, parfois de gombos (mafé); sauce aux oignons et au poulet (yassa); sauce à la viande, au poisson, aux gombos et 
à l'huile de palme (soupou kandia); sauce à la viande, à la tomate et aux légumes (thiou). Les douze personnes interrogées ont toutes consommé un ou plusieurs de ces plats pendant leur séjour à Mantes-la-Jolie.

Quelquefois, on prépare des plats typiques de la moyenne vallée du fleuve Sénégal, des plats consommés aujourd'hui très rarement dans cette partie du pays, comme le gniri, une préparation à base de céréales qui peut être accompagnée de poisson séché, de poisson frais ou de viande. C'est le cas de cette famille habitant le quartier des Garennes, dont la femme âgée de 45 ans est arrivée en France en 1981 (le mari est arrivé en 1975). Elle soutient qu'elle prépare ce plat dans ses deux versions, gniri é bouna et gniri thinklé : "Dans mon ménage, nous aimons bien ces plats qui sont propres aux Peuhls. Je prépare donc les deux. Nous sommes une famille de cultivateurs. Nous avons naturellement gardé nos habitudes alimentaires. Et puis, étant donné que les amis et les parents viennent souvent manger chez nous, ces plats, jen prépare souvent."

La référence aux origines rurales et agricoles pour justifier le maintien des traditions alimentaires est aussi récurrente. Elle rappelle les conditions dans lesquelles la migration masculine en direction de la France s'est opérée. La sécheresse des années soixante-dix, qui a durement déstabilisé l'économie agraire de la vallée, a conduit les hommes à partir pour trouver des ressources nécessaires à la survie des économies domestiques.

\section{Une hybridation timide de l'alimentation du soir}

Les repas du soir sont mixtes. Le repas peut être un plat sénégalais ou appartenant à la cuisine appelée aujourd'hui "internationale". Il existe des plats spécifiques pour la soirée : plats de couscous de mil ou plats à base de céréales et de lait. Les plats à base de couscous varient en fonction des sauces qui les accompagnent : sauce à base de tomate et de viande (sime), sauce à base de tomate, de viande et de beurre de cacahuètes (bassi). Mais l'accompagnement du couscous le plus prisé est le hako. Les feuilles de niébé en sont le condiment de base. Le niébé est une légumineuse à cycle court qui produit une variété de haricos. Les feuilles de niébé sont bouillies. Puis, on y ajoute du poisson séché, des morceaux de viande et/ou des morceaux de poisson frais, de la farine d'arachide et des épices. Ensuite, on sert la sauce obtenue avec du couscous de mil ou de maïs. Toutes les femmes interrogées affirment préparer ce plat qui est l'emblème de l'alimentation pular. Six des douze personnes ayant séjourné à Mantes-la-Jolie ont soutenu avoir mangé du laciiri é hako. Mieux, certains ont reçu à la fin du repas un bol de couscous au lait frais. C'est là une marque distinctive du 
consommer pular dans sa plus pure tradition. En effet, dans la vallée du fleuve Sénégal, c'est par ce bol de couscous au lait frais que se clôture le repas.

Le repas peut comporter des granulés de céréales de mil cuits à la vapeur, accompagnés de lait caillé et sucré (thiakry) ou de pâte à base de céréales recouverte d'une couche de lait caillé (gniri é kossam). Ces plats sont prisés par les ménages de migrants parce qu'ils sont légers et conviennent donc pour le repas du soir. En lieu et place de ces repas sénégalais et du terroir, les familles consomment également une cuisine internationale : poulet et frites, pâtes, pizzas. Ces repas présentent l'avantage d'être préparés rapidement, et surtout d'être très accessibles. On observe quand même quelques emprunts aux traditions culinaires locales. Beaucoup de femmes ont affirmé préparer quelquefois du hachis parmentier.

\section{Les sociabilités autour de l’alimentaire}

Le respect des pratiques alimentaires passe également par les égards témoignés aux arrivants dans un village. La plupart des douze personnes interviewées ayant séjourné à Mantes-la-Jolie étaient, au moment de leur séjour, des fonctionnaires de l'État du Sénégal : enseignants d'université, de collèges ou d'écoles primaires, journalistes et animateurs. D'autres étaient des étudiants. Onze d'entre eux ont été honorés dans la pure tradition du Fouta. Dans cette tradition, honorer un hôte, c'est lui envoyer des plats préparés. On retrouve, parmi les plats envoyés, les plats traditionnellement servis au Sénégal lors des repas de midi (repas à base de riz) et quelquefois le repas servi au dîner dans la vallée du fleuve Sénégal : le lacciri é hako. Cependant, le plat cuisiné le plus souvent et envoyé aux hôtes est le riz au poulet. Cette façon d'honorer l'hôte remplit une fonction sociale et religieuse. L'envoi d'un repas témoigne de son attachement et de son affection. Cela permet d'entretenir des liens et de vivifier les relations. Sur le plan religieux, venir en aide aux visiteurs et aux voyageurs est quelque chose de sacré, car ils sont considérés comme étant vulnérables. Dans la tradition pular, quand on quitte sa maison, sa famille et son village pour s'installer ailleurs, on devient un toumouranké. Ce concept exprime la vulnérabilité. En venant en aide à ces personnes vulnérables, on accomplit des actions religieuses pleines de grâce et de vertu. Analysé sous cet angle, l'envoi d'un repas à un hôte est une marque de dévotion. Arjun Appadurai soutient que l'alimentation permet de cimenter les relations entre, d'une part, les individus et les dieux et, d'autre part, les individus eux-mêmes ${ }^{(2)}$.

On mange souvent assis sur une natte, le bol posé au milieu, comme au Fouta. On peut manger avec la main ou utiliser une cuillère. Les personnes ayant séjourné à 
Mantes-la-Jolie retiennent que, très souvent, les repas sont partagés en fonction du sexe et de l'âge : un bol pour les enfants, un bol pour les hommes adultes et un autre pour les femmes. C'est le même principe au sein des unités de consommation dans les villages d'origine. Cependant, on peut aussi manger à table, avec des assiettes. Mais c'est assez rare, surtout lorsque la famille reçoit des invités.

\section{Faire face aux contraintes d'approvisionnement}

En s'installant en France, les migrants ont amené avec eux leur culture alimentaire. Mais la reproduction sur place des pratiques culinaires et alimentaires a posé et continue de poser des problèmes auxquels sont apportées des solutions allant de l'adaptation à l'innovation. Ces problèmes sont résumés par cette femme de 45 ans originaire du village de K., arrivée en France en 1981 et vivant dans le quartier des Garennes : "Pour certains plats spécifiques, il peut se poser un problème d'approvisionnement en produits exotiques. Il y a certains produits qu'on ne trouve pas ici. Il faut aussi retenir que ce sont des repas dont la préparation exige beaucoup de temps et de travail. Comparés aux plats de la gastronomie française, la différence est énorme. La cuisine sénégalaise nécessite aussi beaucoup d'espace. La façon de faire la cuisine diffère."

La première contrainte révélée ici est relative à l'approvisionnement en produits nécessaires à la préparation des repas. En fonction des plats préparés, ces produits sont le poisson frais, les divers poissons fumés, le beurre de cacahuètes, la farine d'arachide, l'huile de palme, les granulés de mil ou de maïs, le gombo et les feuilles de niébé fraîches ou séchées, etc. Pour les boissons, les produits de base sont le bissap et le pain de singe, etc.

Au niveau de Mantes-la-Jolie, le principal centre d'approvisionnement est géré par M.D., un migrant d'origine sénégalaise arrivé en France en 1974. Il tient

Les femmes peuvent aussi s'approvisionner auprès de commerçants arabes, chinois ou pakistanais au centre commercial de Mantes II ou à proximité de la station de métro Château-Rouge à Paris. dans le Val-Fourré la boutique Colobane, dont le nom rappelle celui d'un célèbre marché aux puces dakarois. Il a débuté son activité en 1983. Il vend tous les produits cités précédemment et se ravitaille soit directement à partir du Sénégal et d'autres pays africains, comme la Guinée pour ce qui est de l'huile de palme, soit à Rungis. D'autres commerçants plus jeunes ont développé une activité commerciale plus structurée. C'est le cas de D.S., migrant 
arrivé du Sénégal en 1994. Il a créé sa société le 4 janvier 2005. Il tient une boutique dans la rue Jean-Jacques Rousseau aux Mureaux où il vend des produits alimentaires. Il dispose aussi de trois camions frigorifiques qui lui permettent de faire des livraisons dans ou hors du département.

\section{Du commerce ethnique à l'innovation}

Les commerçants sont confrontés à des problèmes relatifs à l'emballage des produits, ainsi qu'à l'absence d'étiquetage et de codes-barres. Selon D.S., il est possible de surmonter ces difficultés "en obtenant des autorités françaises des agréments et une normalisation claire, car ces autorités détruisent systématiquement tous les poissons secs pour des raisons d'hygiène".

Les femmes peuvent aussi s'approvisionner auprès de commerçants arabes, chinois ou pakistanais au centre commercial de Mantes II ou à proximité de la station de métro Château-Rouge à Paris.

Pour l'essentiel, la clientèle attitrée de nos deux commerçants est constituée de migrants provenant de leur terroir. C'est pourquoi on peut emprunter à Françoise Lestage son expression de "commerce ethnique pour une demande interne ${ }^{(3)}$ ". Cette qualification d'un commerce tenu par des Mexicains vivant en Californie et vendant des produits mexicains à des migrants d'origine mexicaine s'applique parfaitement ici. Enfin, toutes les femmes interviewées effectuent régulièrement des séjours au Sénégal.

Il peut arriver qu'un condiment assez rare soit en rupture. C'est le cas des feuilles de niébé. Les feuilles, fraîches de préférence, ou séchées, demeurent le condiment de base du laciiri é hako, le plat traditionnel servi au dîner dans toute la vallée du fleuve Sénégal. Lorsque les feuilles de niébé viennent à manquer, certaines femmes les remplacent par des épinards. Cette substitution est culturellement acceptable, car, même au Sénégal, lorsque les feuilles de niébé deviennent rares, on utilise à la place des feuilles de niébéday, un arbre poussant un peu partout au Sénégal.

\section{Les contraintes relatives à la gestion du temps et de l'espace}

Dans la vallée du fleuve Sénégal, l'habitat est fait de vastes concessions dans lesquelles un espace dénommé "kaatané" joue le rôle de "coin cuisine". Si le foyer, centre du kaatané, a une localisation précise, le kaatané demeure un espace qui n'a 
pas de délimitation fixe. On peut vanner ou piler le mil à un mètre ou dix mètres du foyer. La configuration des appartements à Mantes-la-Jolie, et de façon générale en France, ne permet pas cette extension de l'espace dévolu à la cuisine. L'habitat est également inadapté pour certaines préparations qui nécessitent le pilage. Le pilage, réalisé avec un mortier et un pilon que l'on fait venir du Sénégal, fait beaucoup de bruit et peut gêner le voisinage. Les ménages habitant au rez-de-chaussée sont ainsi plus favorisés, car ils peuvent utiliser le mortier sans gêner le voisinage. Ceux qui vivent à l'étage utilisent des astuces pour diminuer le bruit en posant le mortier sur un tapis. Par souci de ne pas déranger le voisinage, cette femme âgée de 50 ans, arrivée en France en 1989 et vivant dans le quartier Godeau, a remplacé le mortier et le pilon par l'utilisation du Moulinex.

Le temps est aussi une contrainte majeure dans la préparation des plats d'origine. Pour des femmes qui ont des occupations professionnelles, préparer des plats sénégalais n'est pas une chose aisée. Selon cette jeune femme de 28 ans, arrivée en France en 2001 (son mari est arrivé en 1989) et habitant dans le quartier Radar, la contrainte de temps est évidente : "Pour cuisiner le laciiri é hako, il faut commencer le matin et compter une demi-journée de travail." 


\section{Adaptation et rationalisation des pratiques}

Les pratiques alimentaires s'adaptent et se rationalisent. Alors qu'au Fouta on envoie au visiteur des plats préparés, ici, très souvent, on envoie aussi des denrées (riz, viande, thé, sucre). Cette adaptation exprime plusieurs choses qui ne s'autoexcluent pas. D'abord, elle évite le gâchis. Si tous les plats préparés étaient envoyés en même temps, ils ne pourraient pas être tous consommés, car certains plats ne peuvent pas se conserver longtemps au froid. C'est l'une des raisons pour lesquelles on envoie des denrées qui peuvent être conservées et utilisées judicieusement. Au Fouta, au contraire, il est exclu d'envoyer des denrées non préparées au visiteur, en lieu et place d'un plat préparé. Ensuite, l'autre raison, tout aussi rationnelle, tient au le gain énorme de temps que l'on réalise en ne préparant pas le repas. En envoyant des denrées au visiteur, on respecte la tradition d'hospitalité, mais, en même temps, on adapte cette tradition à un environnement dans lequel la gestion du temps est un principe directeur.

\section{L'alimentation comme marqueur de l'identité}

Pour cette femme de 50 ans habitant à Godeau, l'alimentation est un puissant marqueur de l'identité : "Nous continuons à préparer des plats sénégalais parce que nous restons des Sénégalais. Le jour où nous cesserons d'être des Sénégalais, nous arrêterons de manger de la nourriture sénégalaise. Manger sénégalais fait partie de notre identité."

Ces propos vont au-delà de l'affirmation de l'alimentation comme composante essentielle de l'ethos sénégalais. Les personnes interrogées expriment l'impossibilité de renoncer à l'alimentation sénégalaise, qui serait synonyme de renoncement à l'identité. Or, dans les représentations des populations pular, renoncer à son identité est quelque chose de très répréhensible.

Lalimentation est aussi perçue comme un vecteur de la transmission de la culture et un moyen d'assurer la cohésion familiale. La jeune femme de 28 ans du quartier Radar s'efforce d'apprendre dans la douceur à ses très jeunes enfants "le vocabulaire alimentaire, la façon de manger, l'appellation des repas et les noms des plats". Elle estime qu'elle le fait pour les éduquer et qu'on transmet aux enfants les pratiques alimentaires pour "pouvoir se reconnaître en eux et être fiers d'eux". De façon générale, les femmes interrogées soutiennent initier leurs enfants à l'art culinaire de leur pays d'origine. 


\section{L'alimentation comme espace de reconstruction d'une mémoire collective}

Un informaticien d'origine pular vivant en Europe raconte une anecdote lors de son passage à Mantes-la-Jolie : une femme de son village l'a hélé du haut du balcon où elle était en train de griller du maïs sur un fourneau (comme on le fait au Fouta), en lui disant : "Viens consommer du maïs." Cet exploitant agricole, qui a été un migrant en France entre 1998 et 2008, a longtemps vécu à Mantes-la-Jolie. Selon lui, les moments de partage des repas entre les hôtes et les invités sont toujours des moments de partage de l'information et de remémoration des histoires communes. Ils offrent l'occasion de parler du village, de son passé, de son présent et de son avenir. On construit une trame historique dans laquelle on vit. En se

\section{Même les commerces} de denrées alimentaires sont des lieux de circulation de l'information et d'entretien du lien social, de la mémoire et de l'identité. remémorant ensemble les souvenirs et les histoires du village et du terroir, on s'entraide, les oublis des uns étant complétés par "la mémoire des autres"(4)". On construit ainsi une mémoire collective. Dans la systématisation des fonctions jouées par l'alimentation, Mintz et Dubois évoquent, en partant des travaux de Sutton, la relation entre celle-ci et le processus social de construction de la mémoire ${ }^{(5)}$. À travers cette mémoire, on refuse "la mort de la distance", pour reprendre les mots de Van der Veer ${ }^{(6)}$. Marc Dedeire et Selma Tozanli écrivent ceci : "Durant son 'frottement' avec la culture d'accueil et la (re)construction identitaire irréversible, le migrant a le réflexe naturel de garder son identité d'origine. Pour le faire, il met spontanément en pratique son savoir-faire qui consiste en la fabrication des objets, des ustensiles, des mets culinaires ${ }^{(7)}$."

Même les commerces de denrées alimentaires sont des lieux de circulation de l'information et d'entretien du lien social, de la mémoire et de l'identité. Le propriétaire de la boutique Colobane estime qu'elle joue un rôle social important au sein de la communauté des ressortissants de la vallée du fleuve Sénégal, au même titre que les associations culturelles et les structures d'accueil, et qu'elle constitue un maillon essentiel du dispositif d'intégration sociale. Il souligne que "les clients se retrouvent et se reconnaissent dans sa boutique. Non seulement c'est un lien avec la culture culinaire du pays mais, aussi un lien avec le pays". 


\section{Conclusion}

À Mantes-la-Jolie, l'analyse des pratiques alimentaires des populations originaires de la vallée du fleuve Sénégal montre l'ancrage de ces populations dans leur culture d'origine. À la suite de Bouly de Lesdain ${ }^{(8)}$, on note que la thèse de convergence qui postule "une uniformisation des pratiques alimentaires" n'est pas vérifiée. Les populations migrantes de Mantes-la-Jolie garderaient jalousement leurs pratiques alimentaires d'origine, signes de leur identité, tout essayant de puiser çà et là des compléments pour assaisonner leur culture alimentaire. Par ailleurs, l'analyse des pratiques conforte la thèse, défendue par Bouly de Lesdain, de la résistance au changement des habitudes alimentaires. En choisissant de s'installer définitivement en France, ces populations ont aussi choisi d'y adapter durablement leurs pratiques alimentaires ou plus simplement leur art culinaire. Comme le note Stefania Capone, "le processus de déterritorialisation se fait rarement sans qu'il y ait reterritorialisation" Le déplacement exige une production qui permette de "réancrer' ce qui a été 'déterritorialisé' dans de nouveaux espaces, réels ou symboliques".

\section{Notes}

1. Isabelle Garabuau-Moussaoui, "La cuisine des jeunes : désordre alimentaire, identité générationnelle et ordre social", Anthropology of Food, Issue 0, April 2001, p. 3. (http://aof.revues.org/index975.htlm).

2. Arjun Appadurai, "How to Make a National Cuisine: Cookbooks in Contemporary India", in C. Counihan, P. Van Esterik (Editors), Food and Culture: A Reader, New York, Routledge, $2^{\text {nd }}$ Edition, 2008, p. 295.

3. Françoise Lestage, "De la circulation des nourritures. La perpétuation des liens sociaux des migrants mexicains via l'approvisionnement en produits alimentaires", Anthropology of Food, S4, May 2008, p. 6. (http://aof.revues.org/index2942.htlm)

4. Maurice Halbwachs, Les cadres sociaux de la mémoire, Paris, Albin Michel, 1994, p. 21.

5. Sidney Mintz, Christine Du Bois, "The Anthropology of Food and Eating", Annual Review of Anthropology, Vol. 31, 2002.

6. Voir Peter Van der Veer, "Transnational Religion", communication au colloque "Transnational Migration:

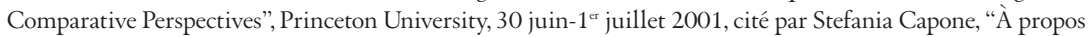
des notions de globalisation et de transnationalisation", Civilisations, ${ }^{\circ}{ }^{\circ}$ 51, 2002.

7. Dedeire Marc et Tozanli Selma, "Les paradoxes dans la construction des identités alimentaires par acculturation", Anthropology of Food, S3, December 2007, p. 2. (http://aof.revues.org/index2582.htlm)

8. Sophie Bouly de Lesdain, "Alimentation et migration, une définition spatiale", in I. Garabuau-Moussaoui,

E. Palomares, D. Desjeux (Éds.), Alimentations contemporaines, Paris, L'Harmattan, 2002.

9. Stefania Capone, op. cit., p. 11. 